\title{
Severe heterotopic ossification following total knee replacement
}

\author{
A Brown, RD Banerjee
}

Robert Jones and Agnes Hunt Orthopaedic Hospital, Gobowen, Oswestry, UK

ABSTRACT

We present a case of severe heterotopic ossification following a primary total knee replacement, which was complicated by a severe intraoperative anaphylactic reaction, and the successful treatment by revision to a rotating platform hinged implant, 32 months following the index procedure.

\section{KEYWORDS}

Heterotopic ossification - Total knee replacement - Anaphylaxis - Stiffness

Accepted 18 February 2018

\section{CORRESPONDENCE TO}

Ashley Brown, E: ashley.brown1@nhs.net

\section{Introduction}

The incidence of heterotopic ossification following total knee arthroplasty (TKA) varies widely across the literature, with rates between $4 \%$ and $42 \%$ reported. ${ }^{1}$ The majority of these cases, however, describe bone formation anterior to the femur, which is rarely of clinical significance. The incidence of severe heterotopic ossification affecting the whole knee and resulting in significant loss of motion is extremely rare and confined to a handful of case reports in the literature.

\section{Case history}

A 76-year-old man with a background of type 2 diabetes mellitus (tablet controlled) and hypertension, who had undergone multiple previous surgeries to the right knee (Table 1), was referred to his local orthopaedic service with right knee osteoarthritis (Fig 1). Preoperative range of motion was 5100 degrees. He underwent an elective primary TKA at a district general hospital.

Intraoperatively, the patient suffered a severe systemic anaphylactic reaction, which was treated appropriately with intravenous adrenaline and hydrocortisone. He was kept intubated and ventilated postoperatively and was successfully extubated 24 hours following the procedure. He underwent routine postoperative rehabilitation and was discharged from hospital seven days postoperatively. He continued outpatient physiotherapy but reports never regaining full extension or flexion. Subsequent tests at a specialist centre did not identify the cause of the anaphylaxis.

At follow-up, his knee was noted to be extremely stiff. Plain radiographs performed five months postoperatively are shown in Figure 2. He underwent a manipulation under anaesthesia eight months following the index procedure by the index surgeon and subsequently an arthroscopic arthrolysis six months later.

The patient was referred to our institution for further management 22 months following the index procedure. At that time, he was complaining of difficulties getting up and out of chairs, walking any distances and a fixed, flexed knee. On examination, he had a fixed flexion deformity of 40 degrees and was only able to flex to 60 degrees. Clinically, both the medial and lateral collateral ligaments had ossified. Plain radiographs are shown in Figure 3.

Blood tests were unremarkable (Table 2). A nuclear medicine bone scan showed increased uptake on both the

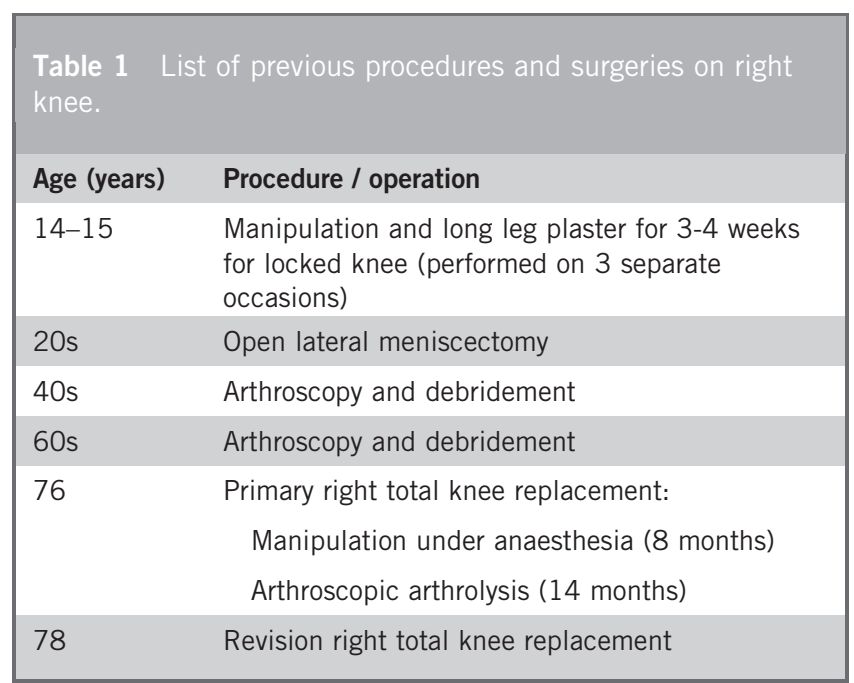




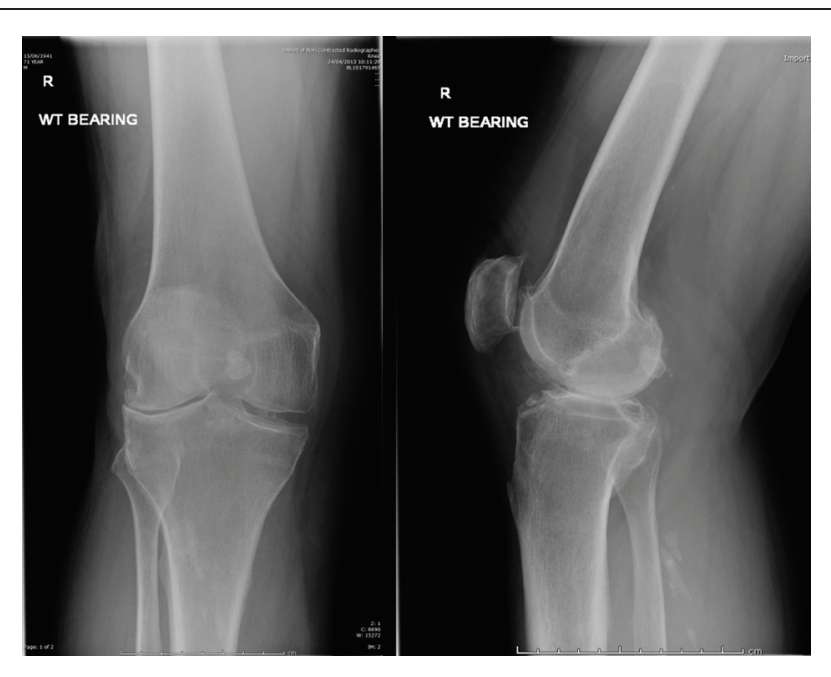

Figure 1 Preoperative plain radiograph of the right knee

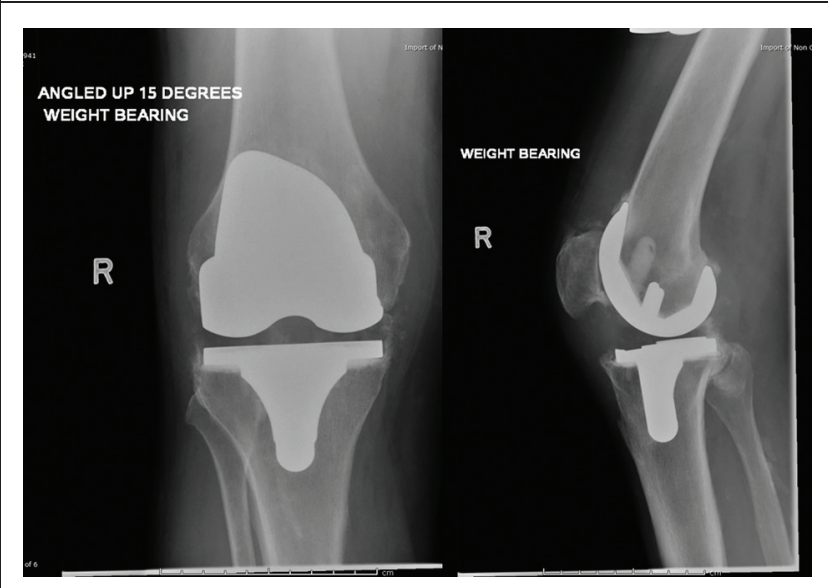

Figure 2 Five-month postoperative plain radiographs showing development of heterotopic ossification

early and delayed phase images globally across the whole of the knee replacement (Fig 4). A knee aspirate was negative for microscopy and culture.

Thirty-two months following the index procedure, the patient underwent revision procedure. The old midline incision was employed, with a standard medial parapatellar approach through thickened scar tissue. Heterotopic bone was removed from around the knee and capsule and a patella tendon peel used to gain access to the joint. There was a large amount of ossification around the posterior aspect of the patella tendon, patella and the quadriceps tendon, and between the femoral component and tibial polyethylene, which was removed sequentially. The medial and lateral collateral ligaments were completely ossified and had to be removed with an osteotome to allow dislocation of the knee. The tibial and femoral components were removed

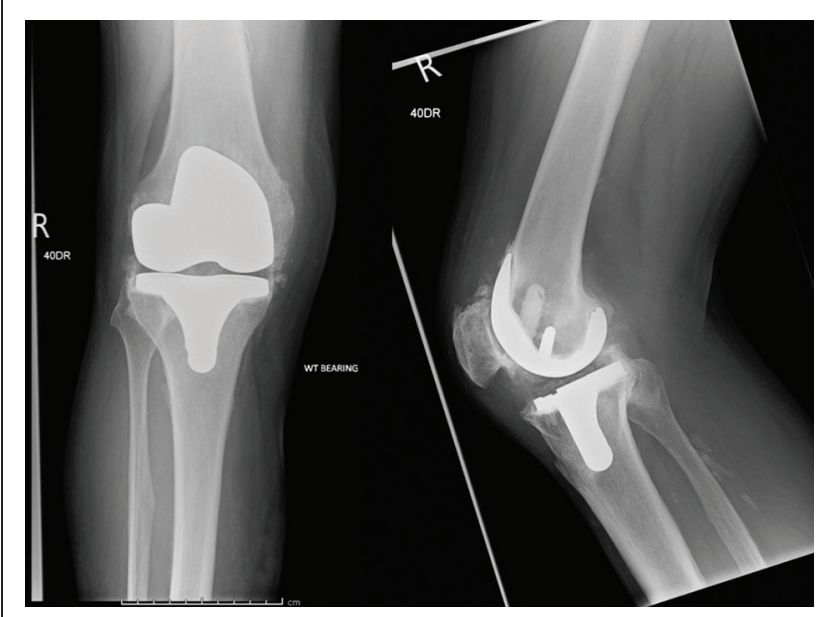

Figure 3 Fifteen months following index procedure, showing established heterotopic ossification (although not applicable to total knee arthroplasty, the pattern of ossification is similar to Brooker 2 for the hip). There is also evidence of lysis around the femoral flange and anterior tibia

\begin{tabular}{|ll|}
\hline Table 2 Bloods results. & \\
\hline Test & Result \\
\hline White cell count $\left(10^{9} / \mathrm{l}\right)$ & 4.9 \\
Haemoglobin $(\mathrm{g} / \mathrm{l})$ & 135 \\
\hline Erythrocyte sedimentation rate $(\mathrm{mm} / \mathrm{h})$ & 10 \\
\hline Sodium $(\mathrm{mmol} / \mathrm{l})$ & 141 \\
\hline Potassium $(\mathrm{mmol} / \mathrm{l})$ & 4.3 \\
\hline Urea (mmol/l) & 6.8 \\
Creatinine $(\mathrm{mmol} / \mathrm{l})$ & 81 \\
\hline Alkaline phosphatase $(\mathrm{iu} / \mathrm{l})$ & 57 \\
\hline C-reactive protein $(\mathrm{mg} / \mathrm{l})$ & 1 \\
\hline
\end{tabular}

without any significant bone loss. Owing to sacrifice of the calcified collateral ligaments, a cemented fully constrained (Stanmore hinge) total knee prosthesis was implanted and the patella resurfaced. On the table, range of motion was -5 to 110 degrees. Intraoperative samples for microbiology were all negative. Postoperatively, the patient underwent a single fraction of radiotherapy (7 Gy) the day following the procedure at a local oncology unit. Indomethacin was not administered because of concerns about interactions with the patient's medications and effect on renal function.

At two weeks postoperatively, his range of motion was 5-65 degrees. Plain radiographs performed at one year postoperatively are shown in Figure 5 . At this point, his range of motion was 10-90 degrees and the patient was extremely satisfied with the result. 


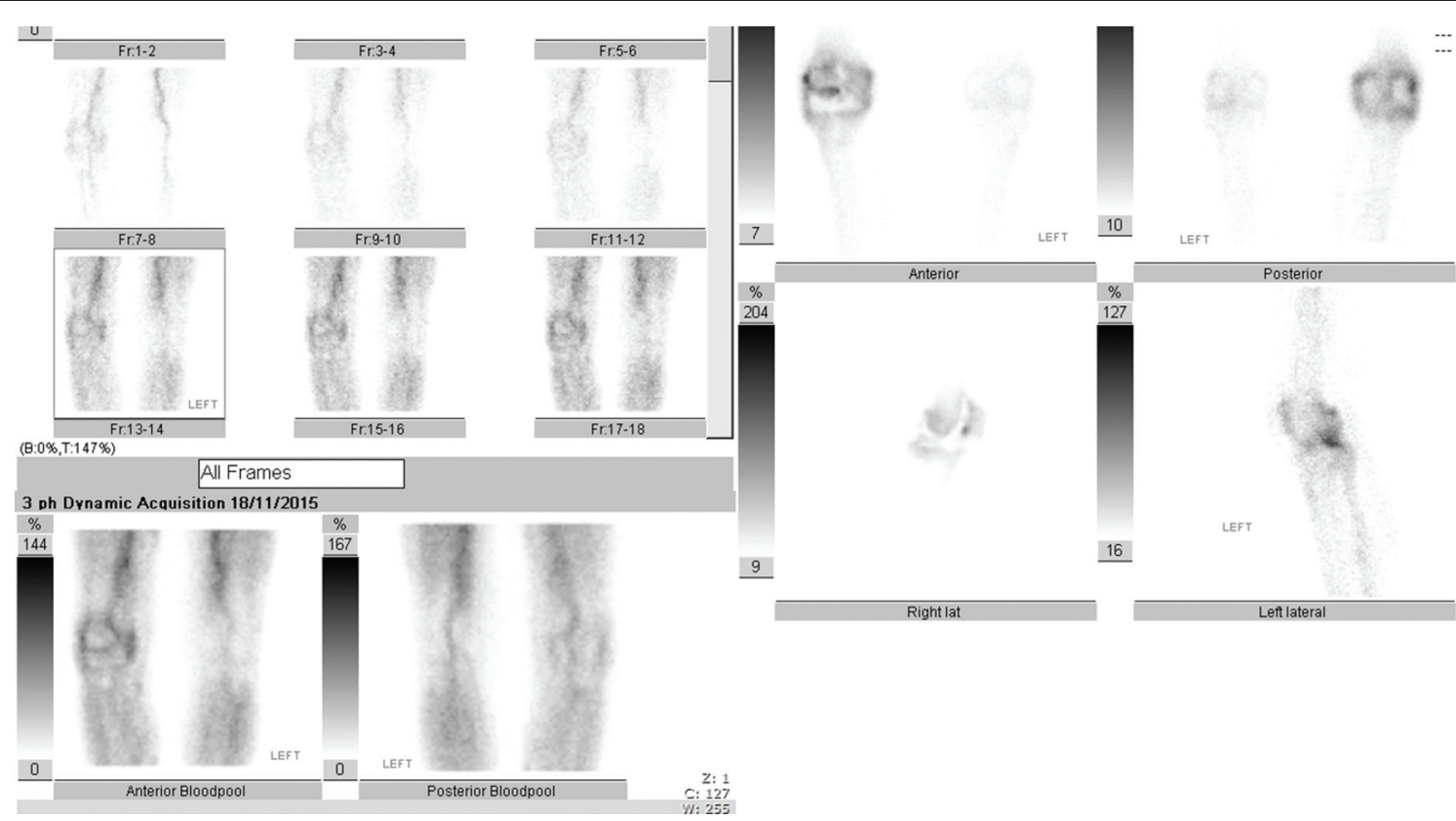

Figure 4 Nuclear medicine bone scan, early (right) and late (left)

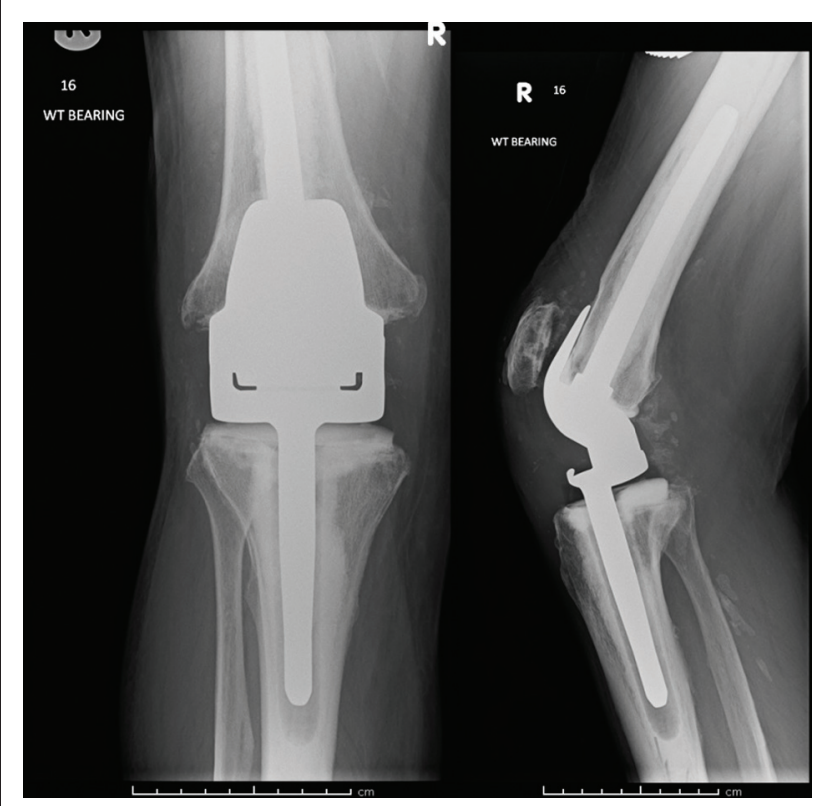

Figure 5 One year following revision with Stanmore hinged prosthesis

\section{Discussion}

The reported risk factors for the development of heterotopic ossification following TKA include gender, ankylosing spondylitis, previous heterotopic ossification of any joint, a history of previous trauma or surgery, previous septic arthritis, hypertrophic osteoarthritis, increased lumbar bone mineral density, anterior notching of the femur, stripping of the periosteum and soft tissues of the distal femur, postoperative haematoma and postoperative manipulation under anaesthesia. ${ }^{2}$

In our case, there were several risk factors for heterotopic ossification, including male sex, previous trauma and surgery, anterior notching of the femur and manipulation under anaesthesia postoperatively. However, this scenario is not uncommon in patients undergoing TKA and such severe heterotopic ossification has not previously been seen at our institution. Another risk factor for postoperative stiffness is soft-tissue stiffness, particularly due to multiple previous surgeries and procedures. Interestingly, the patient also suffered from severe intraoperative anaphylaxis requiring adrenaline and prolonged intubation. There are no reports in the literature of an association between anaphylactic reaction and heterotopic ossification.

Thienpont et al..$^{3}$ report a similar case in a 56-year-old man with lateral compartment osteoarthritis, who underwent TKA, which was subsequently converted to a hinged prosthesis due to severe heterotopic ossification. Prophylaxis against heterotopic ossification was undertaken with one session of radiation therapy ( $7 \mathrm{~Gy}$ ) the day before surgery, as well as oral indomethacin for six weeks. At two years postoperatively, range of motion was 0-115 degrees and no heterotopic ossification was visible on plain radiographs.

The use of a rotating-hinge total knee replacement for treatment of severe heterotopic ossification has only been reported once in the literature, as discussed above. ${ }^{3}$ 
However, it has been reported for the treatment of severe, idiopathic arthrofibrosis following total knee arthroplasty. ${ }^{4}$ In a case series of 14 patients, Farid et $a l .^{4}$ reported on the use of preoperative, low-dose (800 rads) irradiation and constrained-condylar or rotating-hinge revision with patients suffering from arthrofibrosis. Nine patients underwent revision with the rotating-hinge prosthesis and had a mean gain in arc of motion of 55 degrees and a mean improvement of fixed flexion contracture of 24 degrees at mean 34-month follow-up (range 24-74 months).

In summary, surgery for severe heterotopic ossification should only be undertaken following appropriate investigations and should only be considered when the heterotopic ossification has fully matured. Attempts to prevent recurrence need to be considered and liaison with local services in advance should be undertaken to ensure availability of radiotherapy in the perioperative period. Revision to a hinged knee replacement can produce good outcomes.

\section{References}

1. Sterner T, Saxler G, Barden B. Limited range of motion caused by heterotopic ossifications in primary total knee arthroplasty: a retrospective study of 27/191 cases. Arch Orthop Trauma Surg 2005; 125(3): 188-192.

2. Austin KS. Symptomatic heterotopic ossification following total knee arthroplasty. J Arthroplasty 1995; 10: 5.

3. Thienpont E, Schmalzried T, Bellemans J. Ankylosis due to heterotopic ossification following primary total knee arthroplasty. Acta Orthop Belg 2006, 72(4): 502-506.

4. Farid YR, Thakral R, Finn HA. Low-dose irradiation and constrained revision for severe, idiopathic, arthrofibrosis following total knee arthroplasty. J Arthroplasty 2013; 28(8): 1,314-1,320. 